\title{
Replication of band structure in an arbitrary wave vector by holographic modulation
}

\author{
Yoshitaka Kurosaka $\odot,{ }^{*}$ Kazuyoshi Hirose, Hiroki Kamei, and Takahiro Sugiyama \\ Central Research Laboratory, Hamamatsu Photonics K.K., 5000, Hirakuchi, Hamakita-ward, Hamamatsu City, Shizuoka 434-8601, Japan
}

(Received 19 January 2021; revised 21 April 2021; accepted 11 June 2021; published 28 June 2021)

\begin{abstract}
Diffraction is a fundamental physical phenomenon that occurs when scattering wave sources are arranged periodically. For example, when $\mathrm{x}$ rays are incident on the common salt crystals, diffraction occurs owing to the periodic structure of the chlorine and sodium atoms. Recent advances in nanotechnology have enabled artificial control of the scattering points even in visible wavelengths. These artificial structures are called photonic crystals and are related to many interesting phenomena. Dispersion relations between wave vector and frequency are called band structures. Similar to the band structures of electrons and holes in crystals, photonic bands for dispersion determine the direction of propagation of light waves by diffraction of the periodic structures. Herein, we introduce band engineering which enables multiple replications of bands in arbitrary wave vectors by holographic modulation. We have already realized a patterned light source named "iPMSEL" as a typical application of holographically engineered photonic band. The iPMSEL has been realized by combining photoniccrystal surface-emitting lasers and holographic engineering. It can emit arbitrary multiple beam patterns and even photographic images from a tiny semiconductor chip without any optical components. In this paper, we study the iPMSEL from the viewpoint of the photonic-band replication and operation mechanism. While this work is based on photonics, the findings can also be applied to other fields of physics concerned with diffraction. We believe that this work provides useful methods with respect to periodic structures within all scale ranges.
\end{abstract}

DOI: 10.1103/PhysRevB.103.245310

\section{INTRODUCTION}

Diffraction is a special case of scattering in which the scattered wave sources are arranged periodically and interfere mutually — constructively or destructively—in specific directions that Feynman pointed out as, "in order to calculate correctly the probability of an event in different circumstances, we have to add the arrows for every way that the event could happen-not just the ways we think are the important ones!" [1]. In other words, grating is the device that changes the direction of light by controlling interference between periodically arranged scattered wave sources. Such periodic scattering structures can be artificially fabricated using nanotechnology and are called photonic crystals (PCs) [2-5]. One of the successful applications of PCs is in surface-emitting lasers. In two-dimensional (2D) PCs, 2D standing waves can be formed via 2D diffraction. A PC surface-emitting laser [6-14] (PCSEL) uses the standing wave condition of the 2D $\mathrm{PC}$ as a resonator; further, as it enables broad-area lasing, high-quality broad-area plane waves can be obtained, resulting in narrow divergence beams.

Directional emissions called photonic bands can be observed that provide useful information about the $\mathrm{PC}$ resonator [15-24]. For example, the so-called band edge at which the

\footnotetext{
*Corresponding author: yoshitaka.kurosaka@crl.hpk.co.jp
}

Published by the American Physical Society under the terms of the Creative Commons Attribution 4.0 International license. Further distribution of this work must maintain attribution to the author(s) and the published article's title, journal citation, and DOI. slope of a photonic band is zero corresponds to the zero group-velocity condition and lasing occurs under sufficient gain. Subsequently, engineering of the band edge is one of the important issues in constructing photonic-crystal lasers. New band edges can be created at the points at which different bands cross each other in a composite PC with superimposed square and rectangular lattices [24]. Thus, the period of the structure can be modified to engineer the photonic bands; particularly, several studies have been performed on introducing the supercell structure whose period needs to be double $[18,19,23,25]$ in order to observe the photonic band below the light line $[18,19,23]$ and to create an automated design of the PC $[21,22]$. From the viewpoint of a period, quasi-PCs have also been studied [26-29]; however, more controllable methods that can create multiple photonic bands in arbitrary wave vectors have not been reported.

Recently, we proposed and evaluated a functional laser source called the integrable spatial phase-modulating surface-emitting laser (iPMSEL), which emits a static twodimensional beam pattern [30-33]. In the iPMSEL, a planewave source and hologram [34] are integrated into an area less than $1 \times 1 \mathrm{~mm}^{2}$. Computer generated holograms (CGHs) have been used as effective tools for generating beam patterns [34]. The detour-phase method is one of the most common techniques for producing binary holograms [35]; a binary window pattern is used with this method and each window is plotted as a two-dimensionally shifted position corresponding to the spatial phase distribution (see Appendix A). By irradiating the plane wave from the laser source, the wave front is spatially modulated, and the desired beam pattern can be obtained. Therefore, we can control interference to obtain a highly sophisticated wave front by utilizing the 


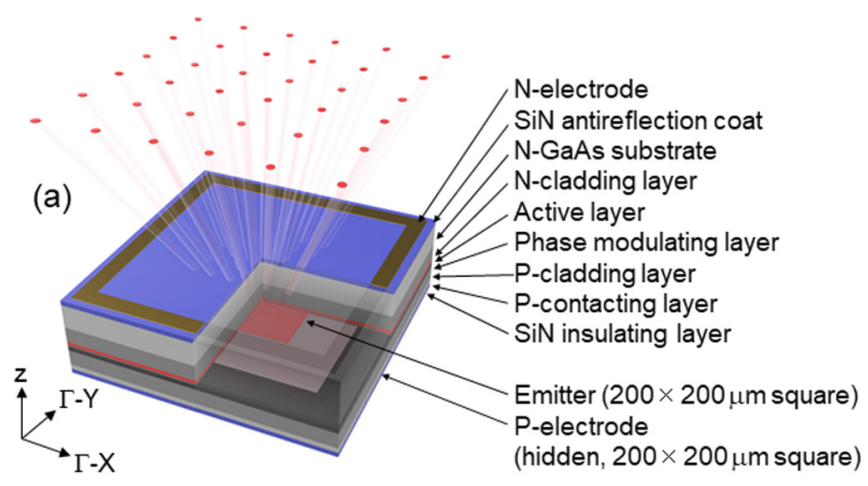

(b)

\begin{tabular}{|l|c|}
\hline Layer & Thickness $(\mathrm{nm})$ \\
\hline N-GaAs substrate & $\sim 200 \mu \mathrm{m}$ \\
\hline N-cladding layer & 2000 \\
\hline Active layer & 185 \\
\hline Phase modulating layer & 270 \\
\hline P-cladding layer & 2000 \\
\hline P-contacting layer & 150 \\
\hline
\end{tabular}

FIG. 1. Device structure of the iPMSEL.

$\mathrm{CGH}$. The iPMSEL uses the quasiperiodic PC resonator as a plane-wave source, based on the PCSEL, and each of its air holes is two-dimensionally shifted holographically, similar to the detour-phase method. When first reported [30], we had not observed the band edge of the complicated beam pattern since there are many bright spots that weaken the diffraction of each spot and hinder the observation of the band edge. Recently, we observed that the photonic band is multiply replicated, and each lasing band edge corresponds to a bright spot, constituting the multispot pattern. Our finding indicates that owing to holographic modulations, photonic bands can be replicated in arbitrary wave vectors, as many as desired.

\section{DEVICE STRUCTURE AND OPERATION MECHANISM}

\section{A. Device structure}

Figure 1 shows the device structure of the iPMSEL. The phase-modulating and active layers are put between the cladding layers; a spatially modulated wave front is formed in the phase-modulating layer and emitted in the vertical direction. Figure 2 shows the phase-modulating layer. Using the target pattern [Fig. 2(a)], we obtained the phase distribution $\phi$ [Fig. 2(b)] corresponding to which each air hole is shifted as shown in Fig. 2(c). Figure 2(d) shows a scanning electron microscope image of the phase-modulating layer where each air hole is slightly shifted from the square lattice. The Fourier spectrum of the phase distribution $\phi$ is shown in Fig. 2(e). The rotational symmetric pattern accompanies the target pattern owing to the in-plane counter propagating standing waves, hence, $6 \times 6$ spot patterns are obtained. This means that the positional shift of air holes changes the wave front such that the $6 \times 6$ spot patterns can be obtained. An additional phase shift as shown in Fig. 3 is introduced to emit the beam pattern vertically because the modulated wave front propagates only in the in-plane directions without the phase shift as mentioned later (see Appendix B and [32,33] for more details). (a)

(b) $\Gamma-Y$

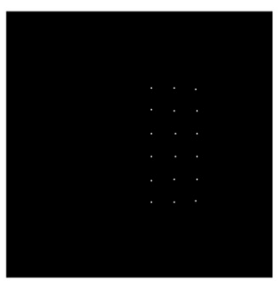

(c)

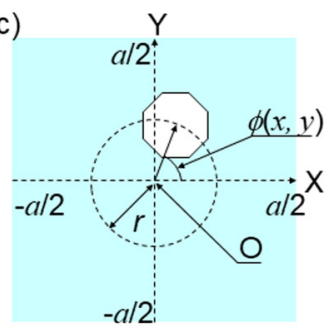

(d)
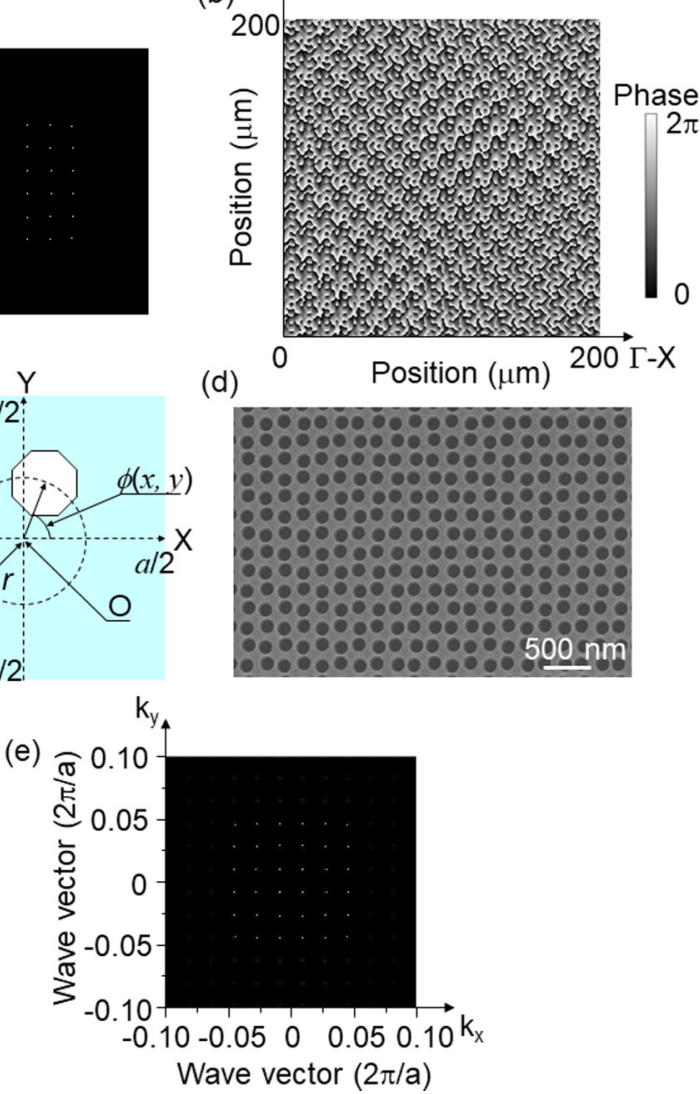

FIG. 2. Phase-modulating layer: (a) target pattern (enlarged view around center), (b) phase distribution, (c) air-hole position in the unit cell, scanning electron microscope image, and (e) Fourier spectrum of phase distribution in (b).

The phase distribution is optimized using the iteration method based on the Gerchberg-Saxton (GS) algorithm [36], and essential information for image construction can be obtained in the phase terms of the complex amplitude. The electro-magnetic field related with the light propagation is described as having a complex amplitude. Therefore, ideally, by reproducing the complex amplitude distribution of the wave front on the device, an arbitrary beam pattern is constructed. In the detour-phase method, the amplitude and phase information are reflected in the size and positional shift of the window, respectively. The phase only hologram is suitable for the unified size of an air hole. We adopt the phase only hologram based on the GS algorithm. This brings a major advantage from the viewpoint of fabrication. Ideally, the beam pattern can be considered as a collection of bright spots. It is clear that the complex amplitude of the multiple spot pattern corresponds to the sum of the complex amplitude of each spot as a result of the superposition principle. As we discuss later, the approach of the superposition of the complex amplitude gives similar results as the holographic approach. Thus, in principle, the holographic approach can be interpreted as a superposition of the diffraction of each spot. However, the above-mentioned holographic approach provides a practical way to generate the arbitrary beam patterns. 


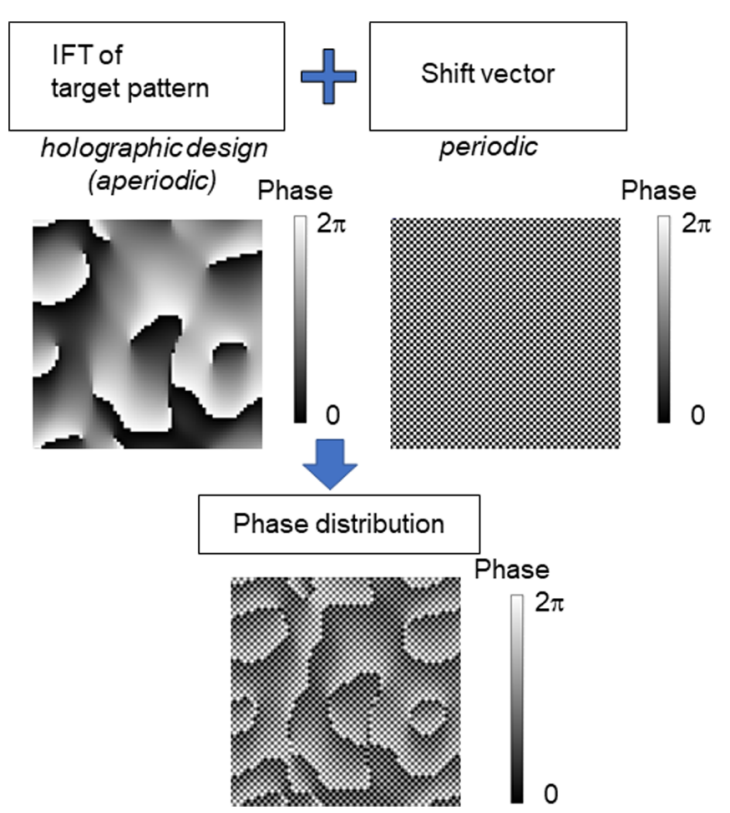

FIG. 3. Concept of phase distribution of the iPMSEL (IFT: inverse Fourier transformation).

\section{B. Operation mechanism}

Figure 4(a) depicts the reciprocal lattice space of a squarelattice PC. The large circles indicate the $\Gamma$ points, and the small circles indicate the $X$ and $M$ points; the $X$ points are the midpoints of the $\Gamma$ points along the $k_{x}$ or $k_{y}$ axis, and the $M$ points are the midpoints of the $\Gamma$ points along the diagonal. Further, the solid line represents the first Brillouin zone [5], and the light line is represented with a dashed circle of radius $k_{\mathrm{LL}}$. Figure 4 (b) represents the photonic band; the dashed line represents the light line which corresponds to the total internal reflection (TIR) condition, and below the light line, all the incident light is reflected owing to the TIR. Therefore, the output light below the light line is not emitted out of the plane directly. The gray area corresponds to the region below the light line. At the light line, the incident angle $\theta_{\mathrm{c}}$ satisfies Snell's law, i.e., $\sin \theta_{c}=1 / n_{\text {eff }}$, where $n_{\text {eff }}$ is the effective refractive index of light. The magnitude of $k_{\mathrm{LL}}$ can be obtained by projection of $k_{\text {air }}$ as $\left|k_{\text {air }}\right| \sin \theta_{c}=\left|k_{\mathrm{LL}}\right|$. Since $k_{\text {air }}=2 \pi / \lambda,\left|k_{\mathrm{LL}}\right|=2 \pi /\left(\lambda n_{\text {eff }}\right)$, where $\lambda$ is the wavelength; therefore, the position of the light line depends on $n_{\text {eff }}$. The band edge labeled " $\Gamma$ " is the $\Gamma$ point used with our early stage device $[30,31]$ and is above the light line; the band edge
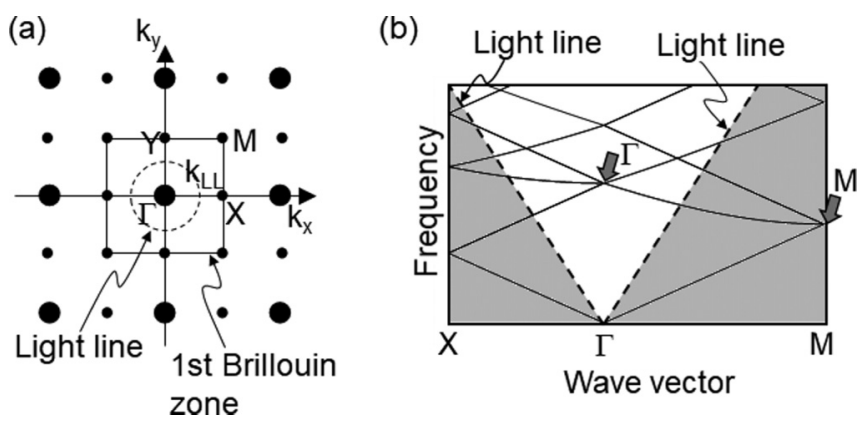

FIG. 4. Light line: (a) light line on the reciprocal lattice space, and (b) light line on the photonic band.

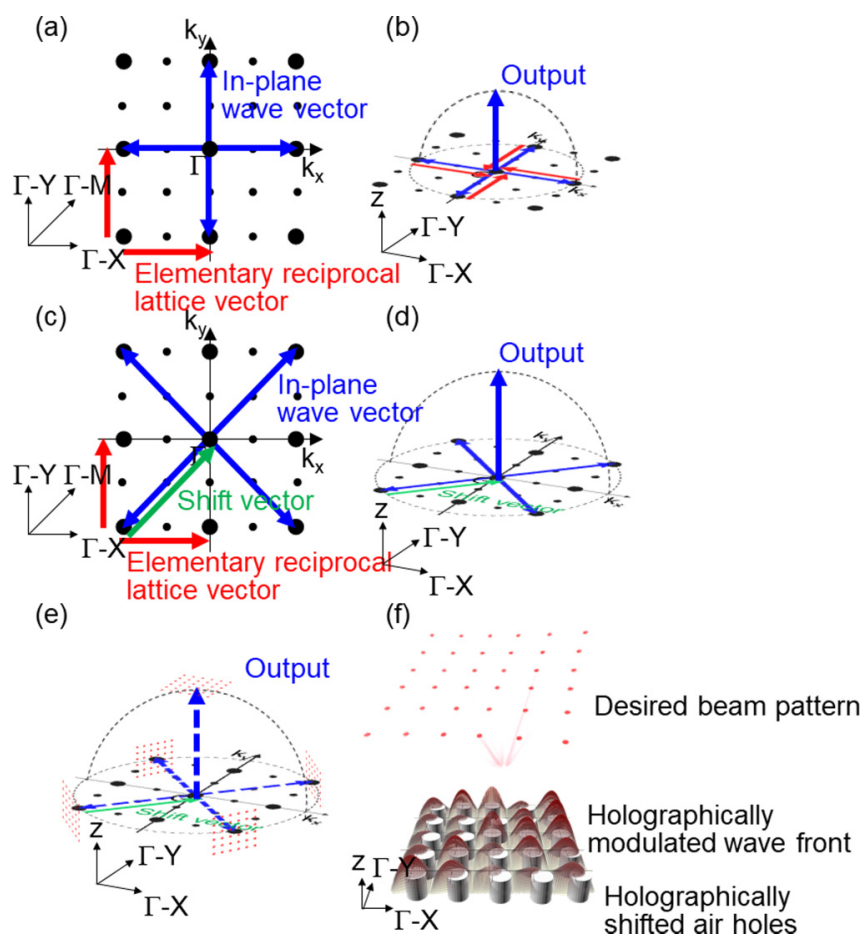

FIG. 5. Concept map of wave front of the square lattice in reciprocal lattice space: (a), (b) $\Gamma$ point of square lattice PC, (c), (d) $M$ point of square lattice PC, (e) $M$ point of the iPMSEL, (f) Intuitive image of the spatial phase modulation of the wave front of the output beam due to positional shift of the holes. The fluctuation of the wave front has been magnified for visibility.

labeled " $M$ " is the $M$ point used with the iPMSEL [32,33] studied herein and is below the light line.

Next, we will discuss as to how the standing wave condition is created at the $\Gamma$ and $M$ point. When a carrier is injected from an electrode, a photon is generated at the active layer. It creates a localized mode in the thickness direction between the cladding layers and a part of the light wave exists on the phase-modulating layer. Therefore, the light wave is scattered by the air holes. Figure 5(a) shows this on a reciprocal lattice space. The four blue arrows indicate the wave vectors of the fundamental light waves propagating in the plus and minus $\Gamma-X$ and $\Gamma-Y$ directions. There are two elementary reciprocal lattice vectors indicated by the red arrows parallel to the $\Gamma-X$ and $\Gamma-Y$ directions respectively. Figure 5(b) exhibits a perspective of the view. As the magnitude of the in-plane wave vectors are equal to that of the elementary lattice vectors, the in-plane magnitudes become zero owing to the diffraction effect of the elementary lattice vector, and they change the direction in the plus and minus $z$ directions owing to the law of conservation of momentum. This corresponds to the diffraction in the vertical direction. In contrast, in case of the $M$ point, as shown in Fig. 5(c), four in-plane wave vectors propagating in the $\Gamma-M$ directions are formed. However, in this case as the magnitude of the in-plane wave vectors and elementary reciprocal lattice vectors are different, the in-plane wave vectors cannot couple to the vertical direction. By introducing the shift vector as a diffraction effect which cancels the in-plane wave vector, a diffraction in the vertical direction occurs [Fig. 5(d)]. 
(a)

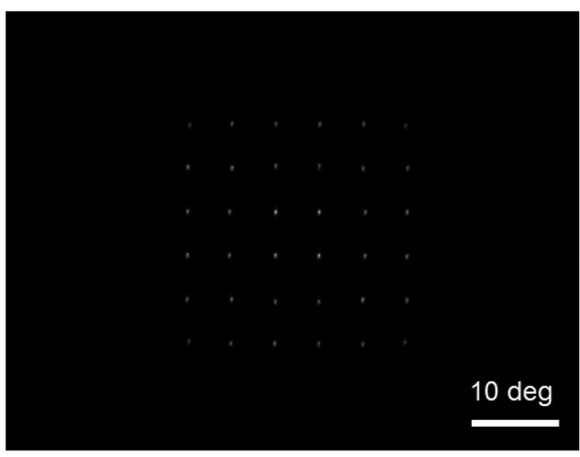

(b)

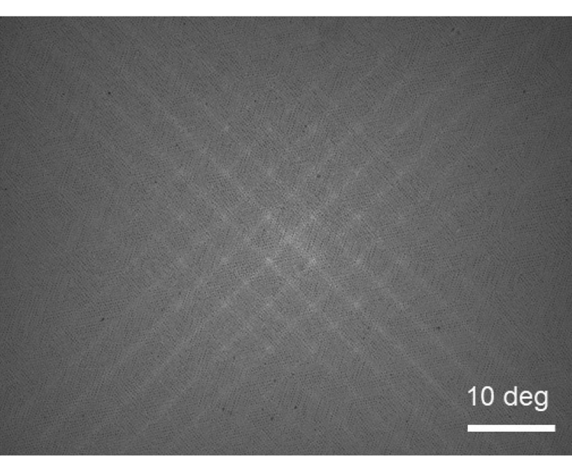

FIG. 6. Far-field pattern: (a) above lasing threshold current (CW $400 \mathrm{~mA}$ ) and (b) below lasing threshold current (CW $320 \mathrm{~mA})$.

The mechanism described above is simplified for ease of understanding. However, in reality, all the air holes are holographically shifted and the vertically propagating wave front is modulated to generate a desired beam pattern without using any optical components as shown in Figs. 5(e) and 5(f) (see Appendix B and [32,33] for more details).

\section{RESULTS AND DISCUSSION}

\section{A. Far-field pattern and photonic band of iPMSEL}

Figure 6 represents the measured far-field patterns of the iPMSEL above and below the lasing threshold current. Above the lasing threshold current [Fig. 6(a)], the $6 \times 6$ spot beams are observed. The iPMSEL emits rotationally symmetric patterns simultaneously [30-33]; therefore, the $3 \times 6$ spot beams [Fig. 2(a)] and their rotationally symmetric patterns are superimposed to produce the $6 \times 6$ spot beams as depicted in Fig. 2(e) (see Appendix B). Multiple X-shaped line patterns below the lasing threshold current [Fig. 6(b)] are observed and such directional patterns could be observed along with the photonic band. Therefore, we measured the directional emission from the iPMSEL below the lasing threshold current (see Appendix C). Normally, the photonic band is obtained by measuring the directional emission from the device (see Appendix C). In principle, the photonic band below the light line cannot be observed. However, as aforementioned, we introduce the shift vector to obtain the vertical diffraction. Therefore, a photonic band below the light line can be observed as a result of the folding of the band.

Thus, we obtained the photonic band shown in Fig. 7(a). Above the lasing threshold current, we obtained the photonic band shown in Fig. 7(b), where the lasing wavelength is highlighted for the same measurement method. By comparing Figs. 7(a) and 7(b), we can determine the position where lasing occurs [16], as each bright spot corresponds to a band edge. Figures 7(c) and 7(d) represent the cross sections at the lasing wavelengths using the dashed lines in Figs. 7(a) and 7(b), respectively. Compared to Fig. 6(a), the bright spots in Figs. 7(b) and 7(d) correspond to the far-field patterns and to Fig. 2(e) as well. The cross sections at other wavelengths are shown in Fig. 8. Surprisingly, multiple replicated photonic bands which are equal to the number of bright spots in the farfield pattern were observed. As aforementioned, each bright spot of the beam pattern corresponds to a band edge. Since the band edge corresponds to the standing wave condition, under sufficient gain these replicated multiple band edges having the same wavelength form the lasing mode to emit the designed image pattern. This is the operation mechanism of the iPMSEL.

\section{B. Photonic bands with different shift vectors}

After this, we study a simple case to confirm the relation between the shift vector and the replication of a photonic band. The following study is discussed only for a simplified understanding. The simplest pattern is the spot pattern placed at the center of the wave-number space, i.e., $(0 \mathrm{deg}$, $0 \mathrm{deg}$ ). A shift vector $\mathbf{V}$ is then introduced to shift the output wave front into the light cone (see [32] or Appendix B). We fabricated the spot-pattern iPMSELs with different Vs. For $\mathbf{V}=(1.00,1.00)[\pi / a]$, the output pattern is a spot beam perpendicular to the iPMSEL's surface (see Appendix B). This shift vector is the normally utilized in the iPMSEL shown in Fig. 3. We have also investigated the iPMSEL with $\mathbf{V}=(0.96,0.96)[\pi / a]$ and $(0.92,0.92)[\pi / a]$. Notably, $\mathbf{V}=$ $(0,0)$ or $\mathbf{V}=(2.00,2.00)[\pi / a]$ corresponds to the case without $\mathbf{V}$, and we cannot observe any patterns below and above the lasing threshold current.

Figures 9(a)-9(c) are the measured photonic bands of the spot-pattern iPMSELs for the direction $(0 \mathrm{deg}, 0 \mathrm{deg})$ with $\mathbf{V}=(1.00,1.00)[\pi / a],(0.96,0.96)[\pi / a]$, and $(0.92,0.92)$ $[\pi / a]$, respectively. In Fig. 9(a), a band edge is observed at the $\Gamma$ point. This iPMSEL was designed to work at the $M$ point of the square lattice; therefore, the plotted wavelength range corresponds to the $M$ point, and there is no band edge. Since the phase of the shift vector $\mathbf{V}$, which has a periodic modulation, introduces another periodicity, a photonic band is observed. Similar to the diffraction in periodic structures, the diffraction in this case can be considered as follows:

$$
\boldsymbol{k}_{d}=\boldsymbol{k}_{i}+\mathbf{V},
$$

where $\boldsymbol{k}_{d}$ is the wave vector of the diffracted light wave, $\boldsymbol{k}_{i}$ is the wave vector of the input light wave, and $\mathbf{V}$ is the shift vector. This suggests that the photonic band can be replicated at the position where the shift in the wave-vector space from the original photonic band is $\mathbf{V}$.

Under the assumption that a holographic shift vector acts similar to the reciprocal lattice vector, we can estimate the position of the photonic band. Then, we compare these experimental results with the theoretical estimates. There 
(a)

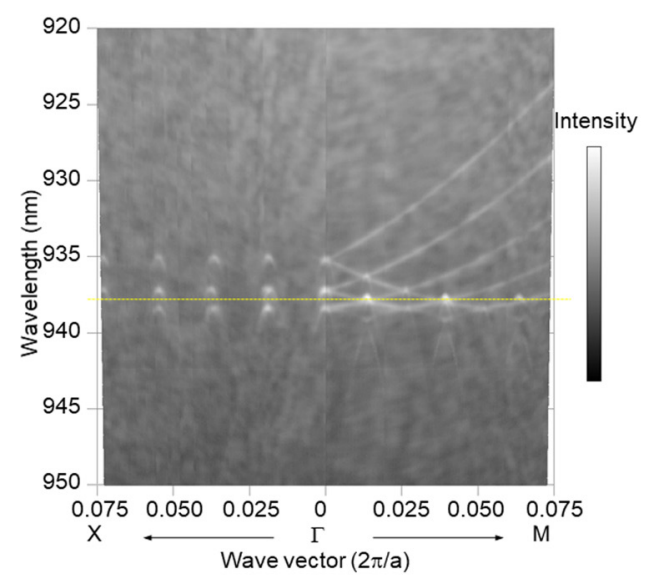

(c)

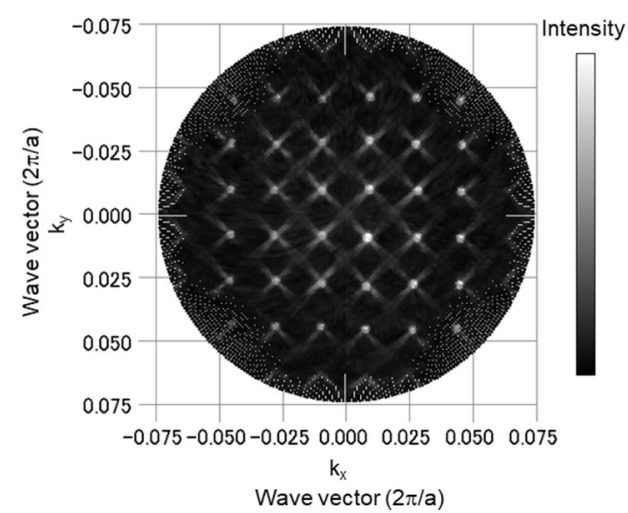

(b)

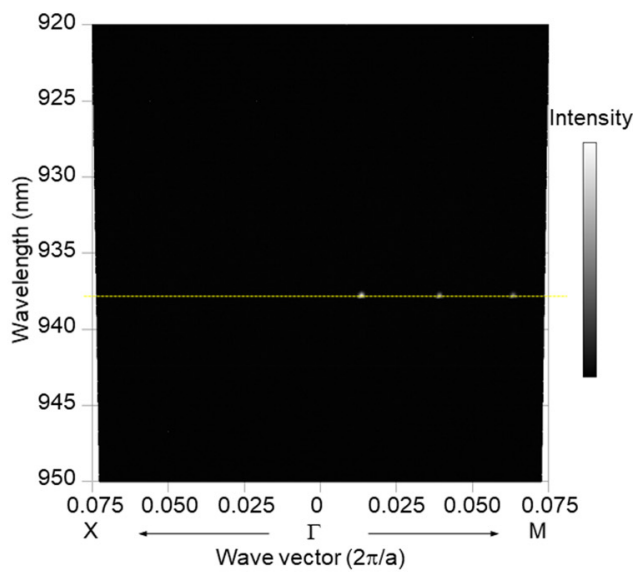

(d)

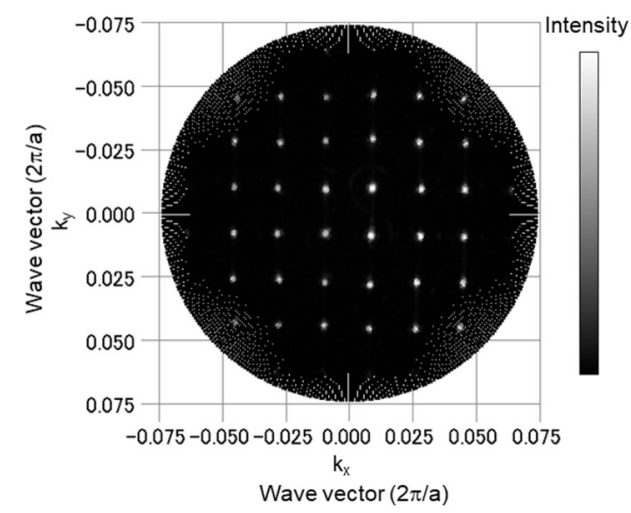

FIG. 7. Measured photonic band of the $6 \times 6$ multispot iPMSEL: (a) cross section along the $X-\Gamma-M$ direction below the lasing threshold, (b) cross section along the same line above the lasing threshold, (c) cross section along the $\Gamma-M$ direction in (a), and (d) cross section at the lasing wavelength (dashed line) in (b).

are several methods for calculating the photonic-band structure. We estimate the wave number of the band edge by calculating the dispersion relation for the square lattice with an infinitesimally small modulation of the dielectric constant (see Appendix D).

Figures 10(a)-10(h) represent the calculated photonic band for an infinitesimally small sized 2D PC lattice modulated with $\mathbf{V}$ values (a) $(0,0)$, (b) $(1.00,1.00)[\pi / a]$, (c) $(0.96$, $0.96)[\pi / a]$, and (d) $(0.92,0.92)[\pi / a]$ [Figs. $10(\mathrm{e})-10(\mathrm{~h})$ are the enlarged views of Figs. 10(a)-10(d), respectively]. The case of $\mathbf{V}=(0,0)$ in Fig. 10(a) corresponds to a normal square-lattice PC. Around the frequency of $0.70[c / a]$, there exists a band edge at the $M$ point [right side of Fig. 10(a)] and no band edge at the $\Gamma$ point [left side of Figs. 10(a) and 10(e)]. However, for $\mathbf{V}=(1.00,1.00)[\pi / a]$ in Fig. 10(b), the folded band (red line) and the original band (black line) are in symmetry, similar as that in $[23,25]$. By comparing Figs. 9 and 10(f)-10(h), we identify the wave vectors of the band edge. This band edge has been folded from the $M$ point and does not originate from the $\Gamma$ point. Strictly speaking, photonic bands that have shifted equal to the integer multiple combinations of the reciprocal lattice vectors are simply superimposed and appear to be a symmetric folded band when plotted in the range of half of the elementary reciprocal lattice vector, i.e., the first Brillouin zone. Therefore, symmetric folding is a special case of shifting. In general, a shifted band structure is simply superimposed and not symmetrical, as shown in Figs. 10(c) and 10(d). Thus far, many studies have reported on the folding and shifting of photonic bands including a supercell structure $[18,19,23,25]$, a composite structure with multiple periods [24], and an automated design of the PC [21,22]. A modulated PCSEL [37] also adds a periodic shift vector to the 2D lattice. Though Fig. 10 is an estimated for an infinitesimally small modulation of the dielectric constant, multiple band edge modes get degenerated. Normally, each band edge mode has a different frequency under a finite dielectric constant difference, as that observed in Fig. 9.

\section{Photonic band replication in arbitrary wave vector}

As a photonic band can be shifted in an arbitrary wave vector by changing the magnitude of the shift vector, multiple replications in an arbitrary wave vector is our interest of focus next. There are two approaches of the CGHs, based on the diffraction theory: the bottom-up and the top-down approaches. The bottom-up approach is to obtain a sum of complex amplitude distribution for each direction based on the idea of angular spectrum [34] which is supported by the superposition principle. Meanwhile the top-down approach is to obtain a multidirectional complex amplitude distribution at once via the idea of diffraction integral, and the GS 
(a)

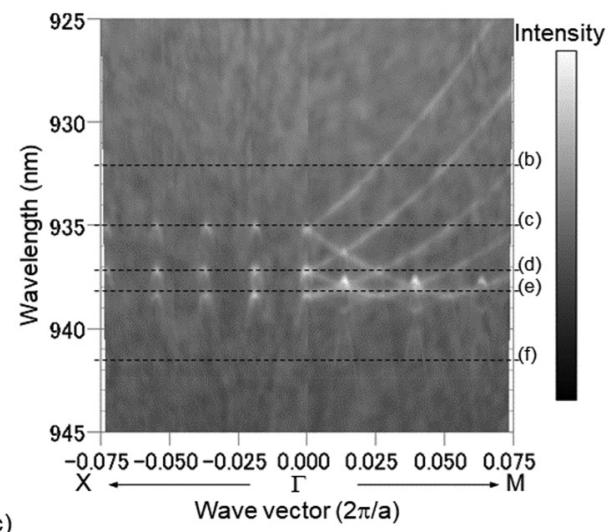

(c)

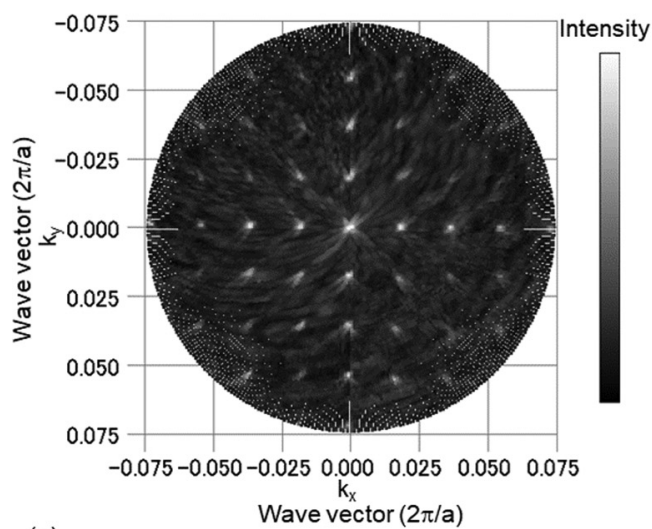

(e)

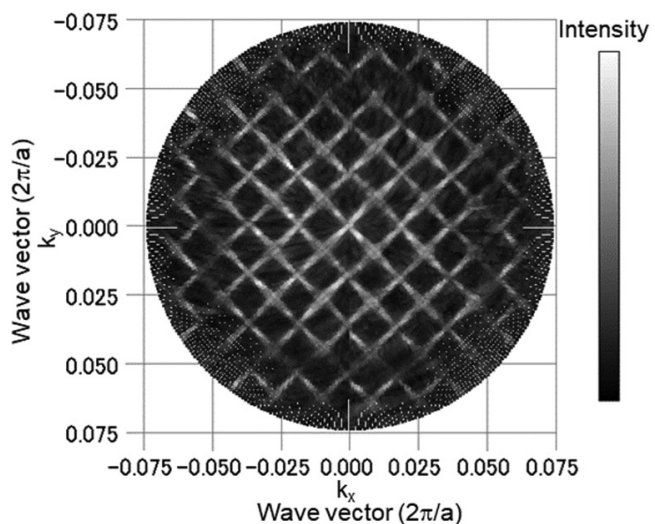

(b)
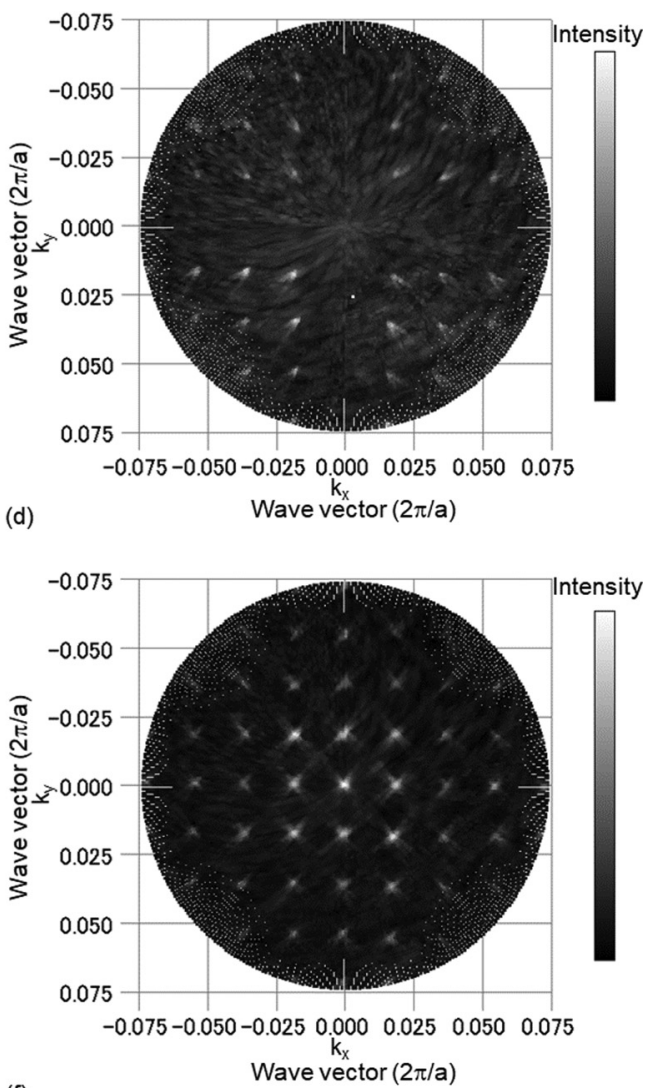

(f)

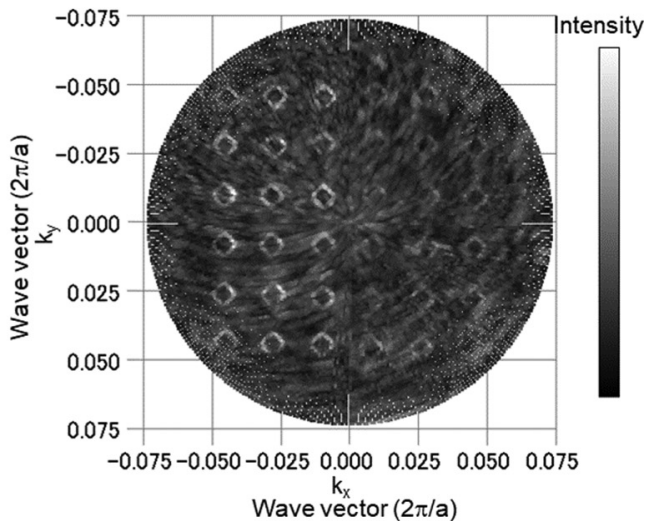

FIG. 8. Cross sections of measured photonic bands of $6 \times 6$ multispot iPMSEL: (a) cross section along the $X$ - $\Gamma-M$ direction below the lasing threshold (CW $320 \mathrm{~mA}$ ), (b)-(f) cross sections at the dashed line in (a) corresponding to 932.02, 935.03, 937.21, 938.21, and 941.46 $\mathrm{nm}$, respectively.

method [36] has been adopted here for usefulness. Although both methods lead to similar results, we have considered the bottom-up approach here because it is intuitive to understand the physics. Since lasing occurs in the band edge of the photonic band, it is possible to realize an arbitrary directional beam. In the case of a single pair beam, the phase distribution is simply periodic [37]. We shall discuss a more general case that consists of more than two periods. The complex amplitude distribution for a single directional plane wave is simply expressed by the periodic function as

$$
A e^{i \phi}=A e^{i k \cdot r}
$$

Therefore, the phase distribution for a single directional plane wave where the wave vector $\boldsymbol{k}=\left(k_{x}, k_{y}\right)$ is described as follows:

$$
\phi=k_{x} x+k_{y} y .
$$

To add the other directional plane wave, the complex amplitude distribution should be simply added based on the superposition principle as follows:

$$
A e^{i \phi}=\sum_{n} A_{n} e^{i \phi_{n}},
$$


(a)

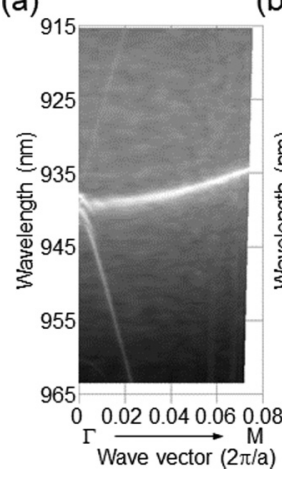

(b)

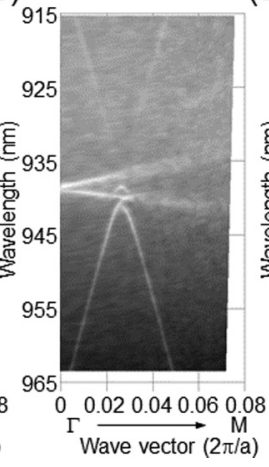

(c)

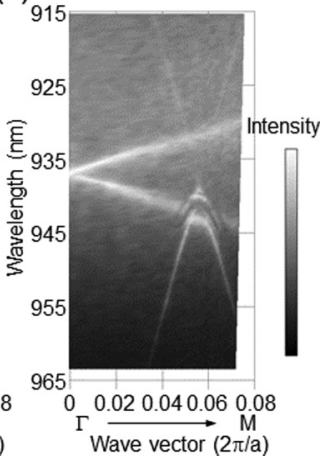

FIG. 9. Measured photonic band of the spot-pattern iPMSEL with different shift vectors $\mathbf{V}$ : (a) $1.00[\pi / a]$, (b) $0.96[\pi / a]$, and (c) $0.92[\pi / a]$.

where $A$ is the amplitude term, $\phi$ is the phase term of the complex amplitude, and $n$ is the number of directional plane waves.

Figure 11 describes this schematically. Figures 11(a)11(c) represent the phase distribution $\phi$ of three different directions. Figures 11(d)-11(f) represent the Fourier transform of Figs. 11(a)-11(c), respectively. The in-plane counterpropagating waves of the standing wave are considered to calculate the beam pattern by substituting the exponential function of Eq. (4) with the cosine function. There is a single pair of spot patterns. Figure 11(g) represents the phase distribution of the sum of the complex amplitude distributions corresponding to Figs. 11(a)-11(c) where the

(a)

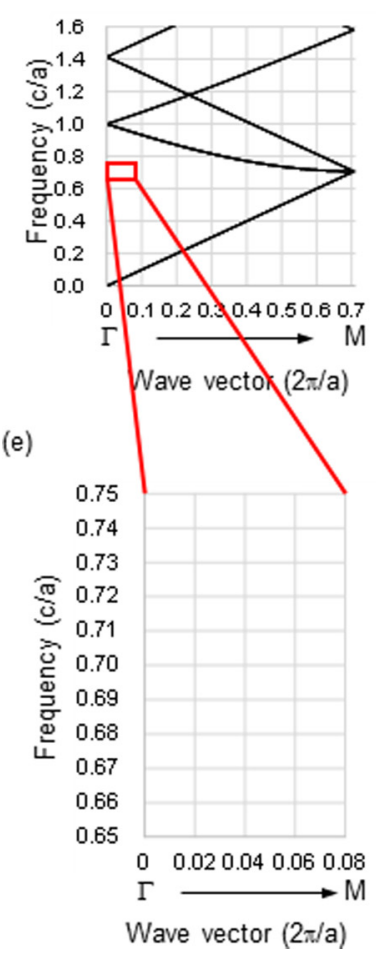

(b)

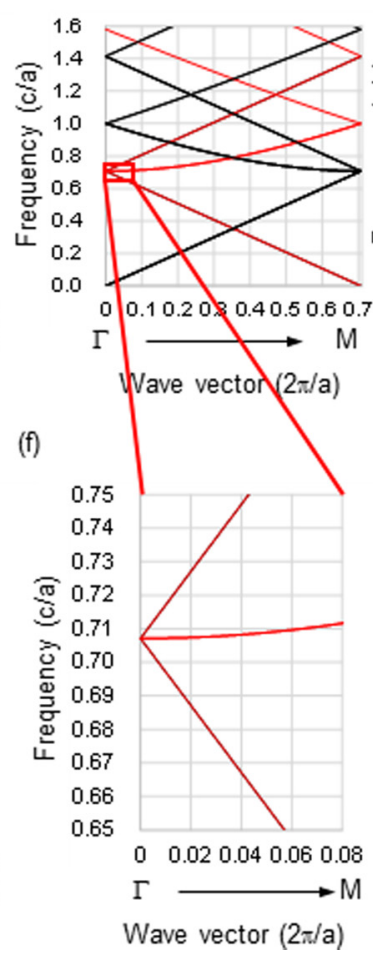

amplitude term $A_{n}$ equals 1 . Figure 11(h) represents the phase distribution obtained using the GS method for which the amplitude distribution is flat. Figures 11(i) and 11(j) represent the Fourier transform of Figs. 11(g) and 11(h), respectively. Comparing Figs. 11(i) and 11(j), the hologram obtained using the GS method gives a similar result, as expected, and thus, it is reasonable to explain the multiple band replication using the idea of the shift vector. Figures 12(a) and 12(b) depict the phase distribution for $3 \times 6$ spot beams, obtained using the superposition of the plane waves and the GS method, respectively. Figures 12(c) and 12(d) represent the Fourier transform of Figs. 12(a) and 12(b), respectively. Also, in the case of a complex pattern which contains many directional waves, a hologram obtained using the GS method is effective in explaining the complex amplitude distribution.

To summarize the band structures, Fig. 13 represents this concept pictorially. In the case of a free space without any air holes [Fig. 13(a)], dispersion relations for the incident plane wave are linear [Fig. 13(b)]. In the case of the 2D lattice [Fig. 13(c)], photonic bands are formed as a result of the shifting of the dispersion relations by the elementary lattice vectors [Fig. 13(d)] (see Appendix D). The shift vector and inverse Fourier transformation of the target pattern work as additional diffraction sources; the former is periodic, whereas the latter contains multiple periods. In the special case of the shift vector in which $\mathbf{V}=(1.00,1.00)[\pi / a]$, air holes are shifted periodically from the 2D lattice [Fig. 13(e)]. Owing to the diffraction effect of the shift vector, the photonic band is shifted in the $\Gamma-M$ direction [Fig. 13(f)]. The black and red lines indicate the original photonic band of the 2D lattice and the replicated photonic band due to diffraction of the shift vector, respectively. In this special case, the shifted

(c)

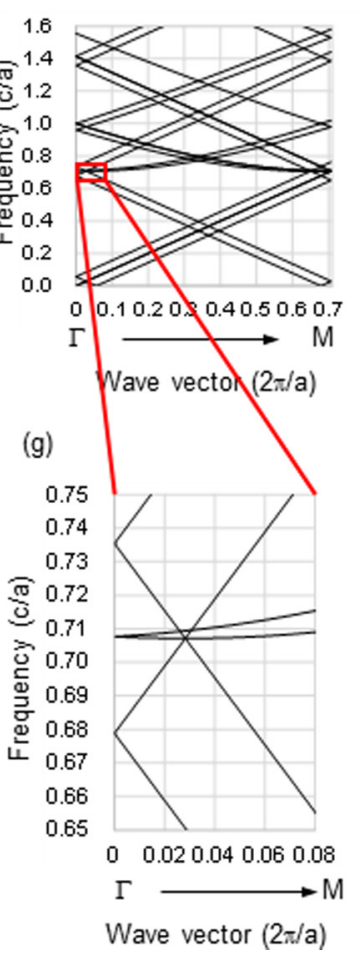

(d)

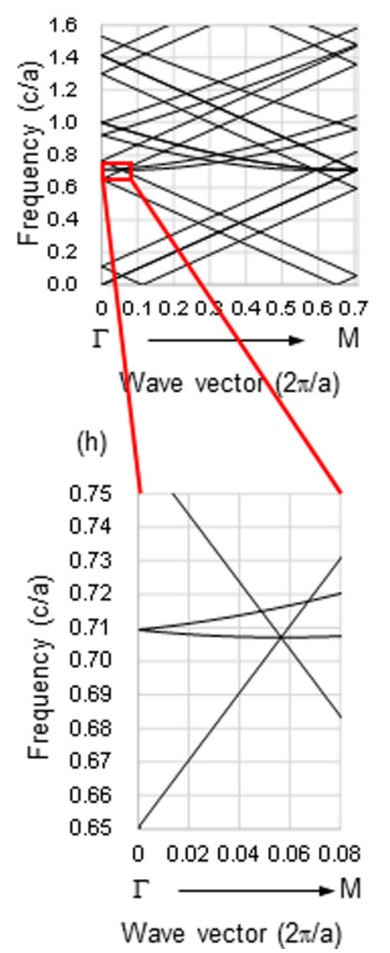

FIG. 10. Calculated photonic bands of infinitesimally small modulations of the dielectric constant with different Vs: (a), (e) (0,0), (b), (f) $(1.00,1.00)[\pi / a],(\mathrm{c}),(\mathrm{g})(0.96,0.96)[\pi / a]$, and $(\mathrm{d}),(\mathrm{h})(0.92,0.92)[\pi / a]$. 
(a)

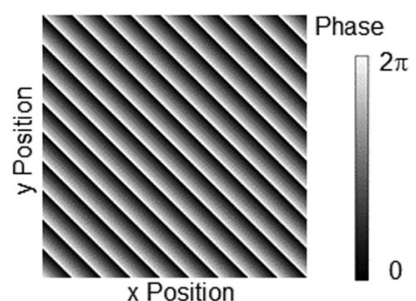

(d)

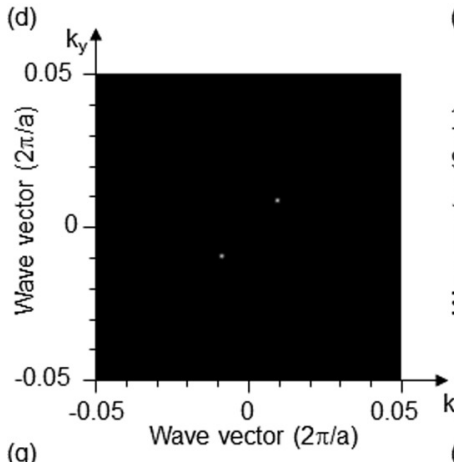

(g)

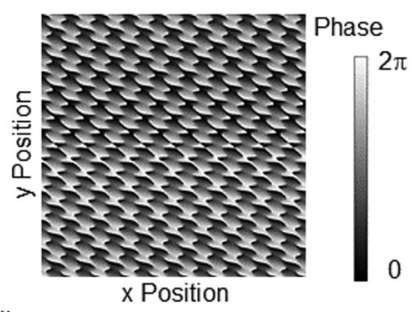

(i)

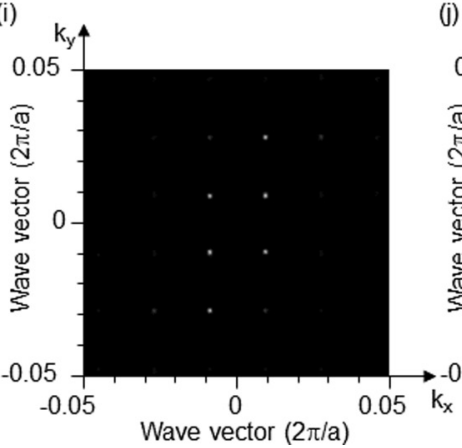

(b)

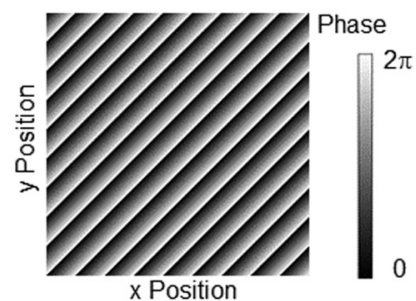

(e)

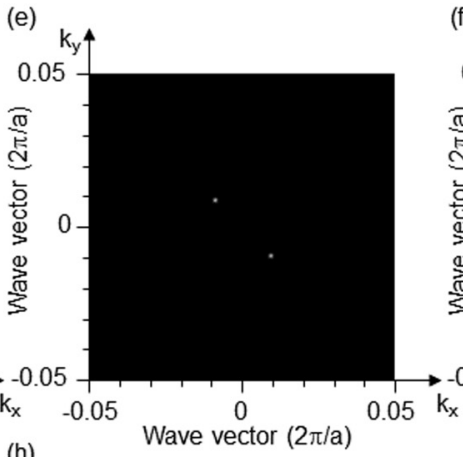

(h)
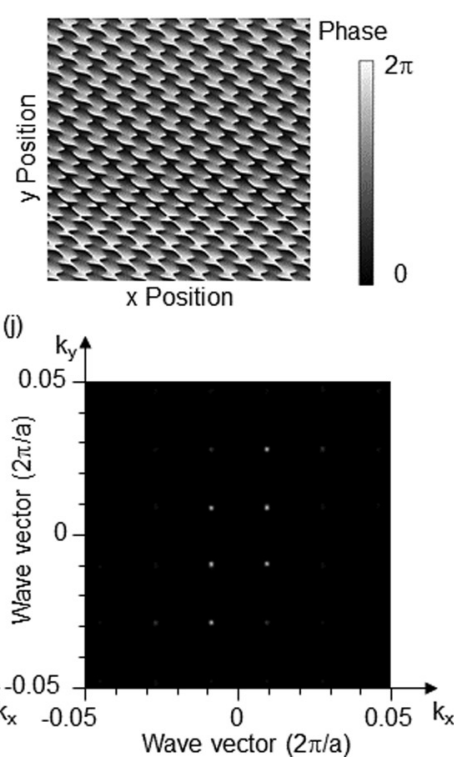

(c)

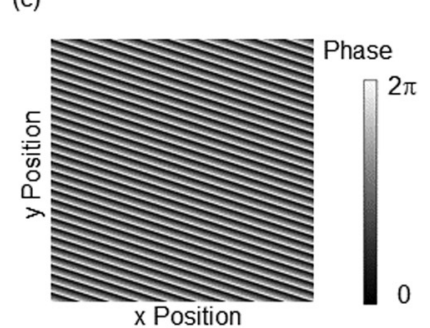

(f)

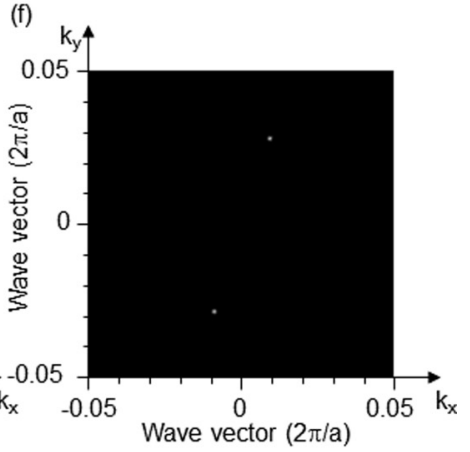

Wave vector $(2 \pi / a)$

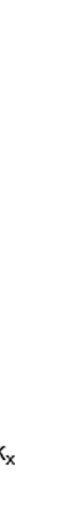


(a)
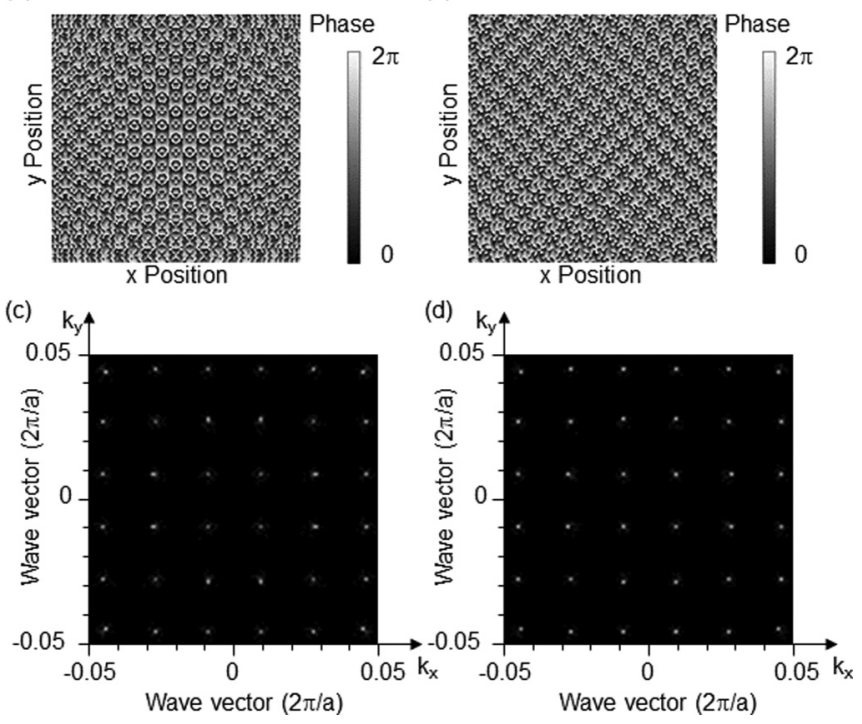

FIG. 12. Phase distribution and corresponding Fourier transform for $3 \times 6$ spot beams. (a) Phase term obtained by the superposition of the plane wave. (b) Phase term obtained using the GS method. (c), (d) Fourier transform of (a) and (b), respectively. Range of Fourier transform is -0.05 to $0.05[2 \pi / a]$ as in Fig. 2(e).

holographic modulations an arbitral number of replications is possible.

Finally, we elaborate as to why we were unable to notice these mechanisms in our previous report [30]. The lasing band edges of each spot were not observed in the previous work [30] because of two reasons. The first reason is that too many spots in the beam patterns weakened the diffraction of each spot, and the second reason is choosing the $\Gamma$-point band edge which accompanies a bright central spot noise which caused a hindrance in observing the band structure of each spot.

\section{CONCLUSION}

We investigated the band structure of the iPMSEL and found the photonic band shifts and replicates using the holographic modulation of the air holes. The photonic band normally shifts because of diffraction caused by the periodic structure; therefore, to shift the photonic band, a different periodic structure should be added, such as a composite PC or supercell structure. However, when the number of periods increases periodic information decreases owing to superposition. From this point of view, holographic modulation provides a different degree of freedom to engineer the photonic band as it can replicate the band structure in an arbitrary wave vector for any arbitrary number. This just enables the working of the arbitrary pattern laser iPMSEL as a practical application [38]. It should be emphasized that this replication approach can be applied not only to PCs but also to other periodic structures which form a band structure. We believe that this finding will enable the engineering of band structures in various fields. (a)

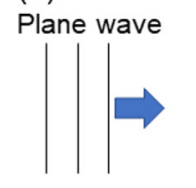

(c)
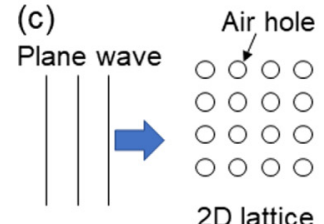

(e)

Plane wave

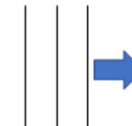

1

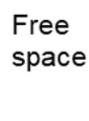

2D lattice
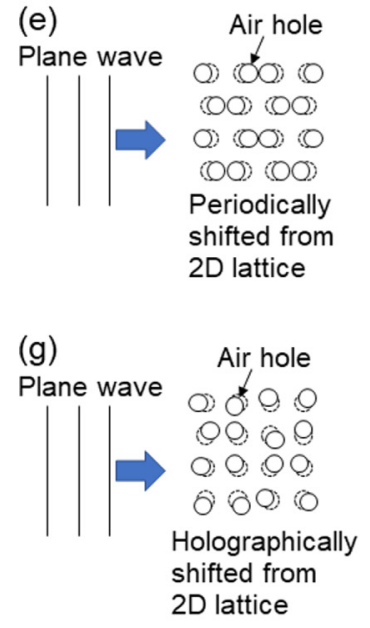

(b)

(f)

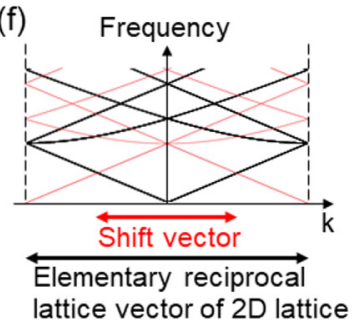

(h) Frequency

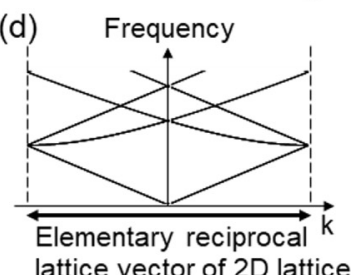

lattice vector of $2 \mathrm{D}$ lattice

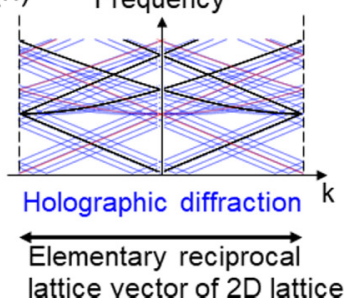

FIG. 13. Schematic of the photonic band: (a) free space without air hole, (b) dispersion relation of free space, (c) air holes arranged in a 2D lattice, (d) photonic band of the 2D lattice (black lines), (e) air holes periodically shifted from the 2D lattice by the shift vector, (f) photonic band of periodically shifted air holes from the 2D lattice by the shift vector (red lines), (g) air holes holographically shifted from the 2D lattice, and (h) photonic band of holographically shifted air holes (blue lines). The plotted range is first Brillouin zone of square lattice.

\section{ACKNOWLEDGMENTS}

The authors are grateful to A. Hiruma (President), T. Hara (Director), M. Yamanishi (Research Fellow), M. Niigaki, Y. Yamashita, K. Nozaki, H. Toyoda, T. Hirohata, T. Edamura, and A. Watanabe of HPK for their encouragement throughout this work as well as A. Higuchi and M. Hitaka for their assistance with the epitaxial growth. This work was partly supported by the Japan Science and Technology Agency (JST); Center of Innovation Program (COI) (Grant No. JPMJCE1311).

\section{APPENDIX A: DETOUR-PHASE METHOD}

The detour-phase method was proposed by Brown and Lohmann in 1966 [35]. A binary window pattern is placed over the 2D cells, and each window is laterally shifted according to the phase of the complex amplitude obtained from the Fourier transform of the target pattern. When a plane wave is irradiated on the 2D window array, a holographically modulated wave front is obtained to form the desired beam pattern. 


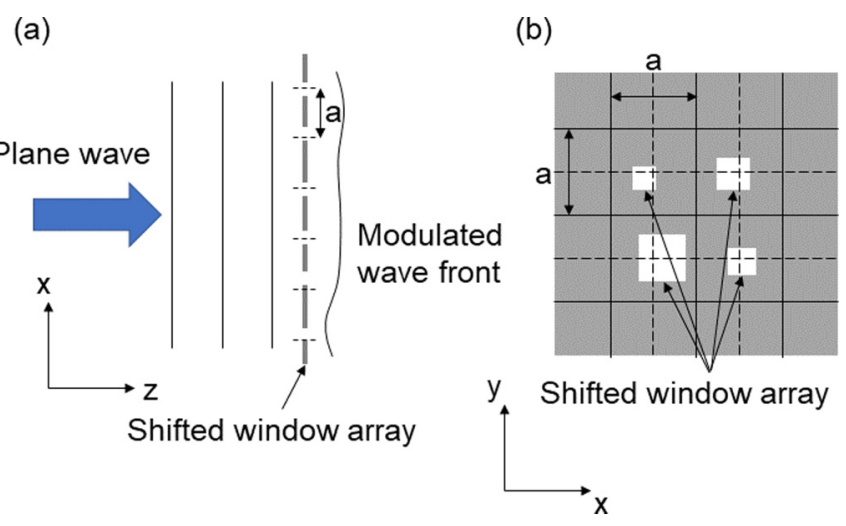

FIG. 14. Concept map of the detour-phase method: (a) cross section perpendicular to the incident plane wave and (b) cross-sectional view.

(For a detailed explanation of the detour-phase method, see [35].) The concept map of the detour-phase method is shown in Fig. 14(a). A shifted window array has many windows through which light can be transmitted. Cells are placed at regular intervals $a$, and the dashed horizontal lines indicate the centers of the cells. Each cell has one window, and each window is shifted corresponding to the phase and size of the window, which in turn is varied corresponding to the amplitude of the complex amplitude distribution. Therefore, when a plane wave is irradiated from the left side of the shifted window array, a modulated wave front is output along the right side. A front view of the shifted window array is shown in Fig. 14(b). Each cell has one window, which is shifted against the center of the cell, corresponding to the cross point of the dashed lines. Using the detour-phase method, the transmissive window pattern and plane-wave source are separated. In the iPMSEL, however, we integrate them by introducing the phase-modulating layer, which enables concurrent lasing and spatial phase change.

\section{APPENDIX B: WORKING MECHANISM AND SHIFT VECTOR OF iPMSEL}

It is reasonable to assume that the in-plane resonance of the iPMSEL is similar to that of the PCSEL since the positional hole shifts are small. Owing to the diffraction of the 2D PC at the $M$-point band edge, four basic in-plane light waves are formed in the diagonal directions, as shown in Fig. 5(c). These basic light waves are coupled and form a 2D standing wave that acts as an in-plane resonance cavity. A surface normal diffraction is prohibited at the $M$-point band edge; therefore, the zero-order beam in the surface normal is removed. In contrast, the scattered light wave at each hole is retarded/proceeds by the local positional shift of the holes, such that the wave fronts of the scattered light waves are locally modulated in accordance with the angle $\phi(x, y)$. As shown in Fig. 3, the phase distribution is obtained using the sum of the original phase distribution $\phi(x, y)$ and the additional phase distribution $\phi_{a}(x, y)$. The additional phase distribution is expressed as

$$
\phi_{a}(x, y)=\mathbf{V} \cdot \mathbf{r}=V_{x} x+V_{y} y
$$

where $\quad x=N_{x} a \quad\left(N_{x}=0,1,2, \ldots\right), \quad y=N_{y} a \quad\left(N_{y}=\right.$ $0,1,2, \ldots)$, and $\mathbf{V}$ is the shift vector.

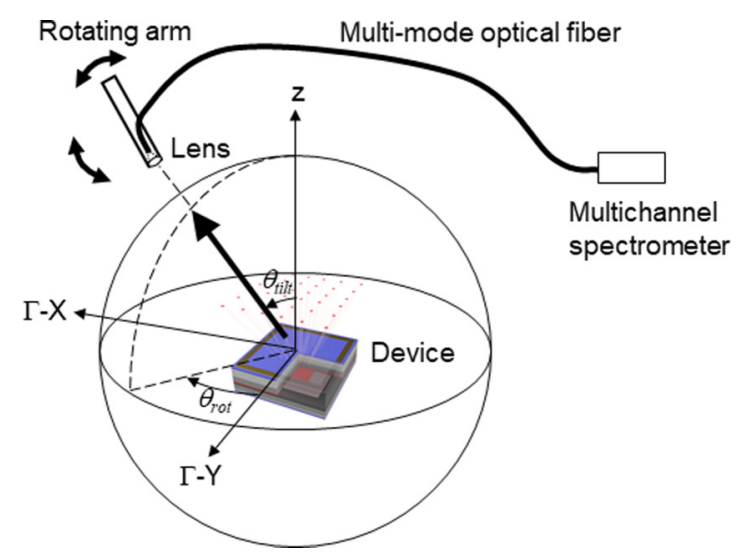

FIG. 15. Setup of the photonic-band measurement scheme.

When $\mathbf{V}$ is defined as follows:

$$
\mathbf{V}=\left(\frac{\pi}{a}\right)\left(\begin{array}{l} 
\pm 1 \\
\pm 1
\end{array}\right),
$$

it completely cancels the in-plane wave-vector component of the basic light waves [green arrow in Fig. 5(c)]. In this case,

$$
\phi_{a}(x, y)=\pi\left( \pm N_{x} \pm N_{y}\right) .
$$

The $\phi_{a}(x, y)$ is a mosaiclike pattern according to Eq. (B3) and tilts the target beam patterns from the direction along the in-plane basic light waves to the surface normal, as shown in Fig. 5(e). Therefore, the total phase distribution is expressed as $\phi(x, y)+\phi_{a}(x, y)$, which enables the projection of the 2D beam patterns out of the plane without the surface normal zero-order beam.

\section{APPENDIX C: SETUP FOR PHOTONIC-BAND MEASUREMENT}

We can measure the photonic band using the directional distribution of the spectra $[15,16,20]$. Figure 15 depicts the setup for the photonic-band measurements. The measurement setup consists of a multichannel spectrometer (HORIBA, iHR320), multimode optical fiber, lens, and rotating arm. Emissions from the iPMSEL in a specific direction were measured using a multichannel spectrometer through a multimode optical fiber. We have defined the spherical polar coordinates around the device. The polar angle $\theta_{\text {tilt }}$ and azimuthal angle $\theta_{\text {rot }}$ have been transformed into the normalized wave vector $\left(k_{x}, k_{y}\right)$ in the reciprocal space as follows:

$$
\begin{aligned}
& k_{x}=(a / \lambda) \sin \theta_{\text {tilt }} \cos \theta_{\text {rot }}, \\
& k_{y}=(a / \lambda) \sin \theta_{\text {tilt }} \sin \theta_{\text {rot }},
\end{aligned}
$$

where $k_{x}$ and $k_{y}$ are the wave vectors parallel to the $\Gamma-X$ and $\Gamma-Y$ directions, $a$ is the lattice constant, and $\lambda$ is the wavelength of light in air. The measurements were carried out under continuous wave operation at the room temperature $\left(25^{\circ} \mathrm{C}\right)$.

To obtain the angular dependence of the device at the lasing wavelength [Figs. 7(c), 7(d), and 8(b)-8(f)], the measurements along each direction were stored in 3D arrays as $\left(k_{x}, k_{y}, \lambda\right)$. Then, a horizontal cross section through this array 


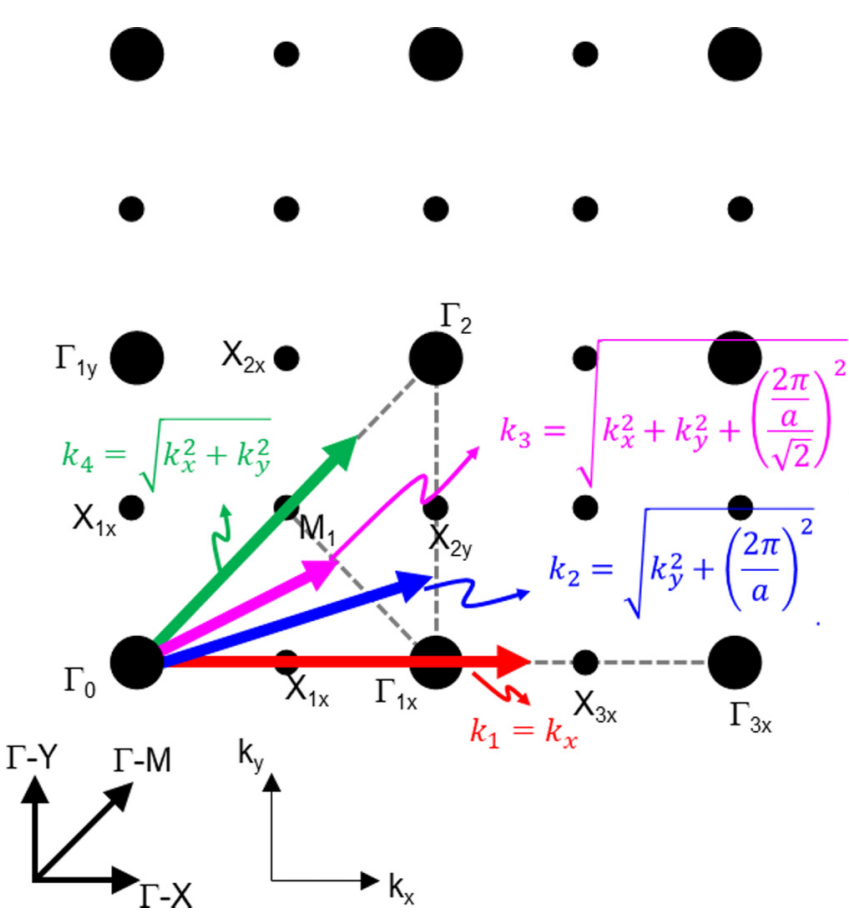

FIG. 16. Reciprocal lattice space of the square-lattice PC.

at a desired wavelength yielded the angular dependence of the device. The color scale indicates the light intensity.

\section{APPENDIX D: DISPERSION RELATION FOR THE SQUARE LATTICE WITH AN INFINITESIMALLY SMALL MODULATION OF THE DIELECTRIC CONSTANT}

To estimate the wave number of the band edge, we calculate the dispersion relation for the square lattice with an infinitesimally small modulation of the dielectric constant [5]. The dispersion relation of a 2D PC with a small spatial variation of the dielectric constant can be represented by the

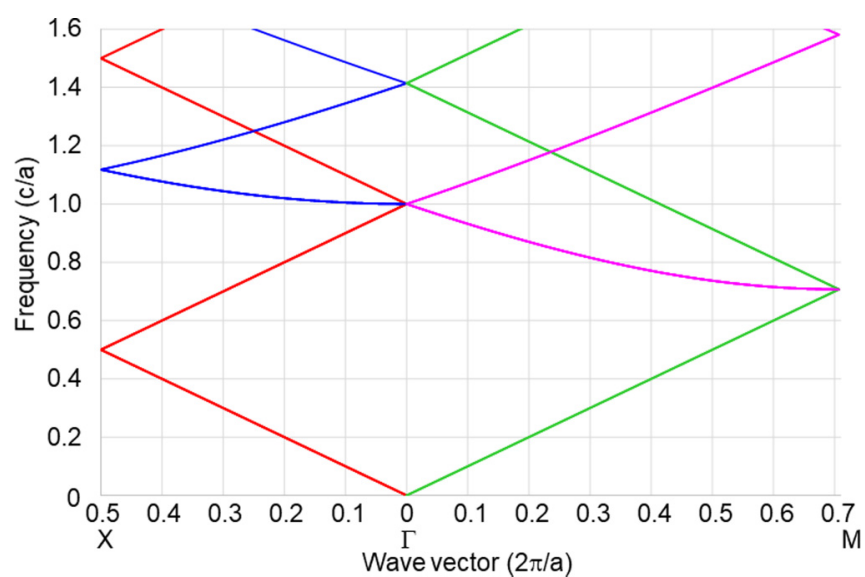

FIG. 17. Dispersion relation for the square lattice with infinitesimally small modulations of the dielectric constant in air.

folding of the dispersion line of the uniform material, $\omega=v k$, into the first Brillouin zone [5]. Figure 16 represents the reciprocal lattice space of a square-lattice PC, where the large circles indicate the $\Gamma$ points and the small circles indicate the $X$ and $M$ points, as illustrated in Fig. 4(a). We plot the dispersion relation along the $\Gamma-X$ and $\Gamma-M$ directions. For example, the dispersion relation along the $\Gamma-X$ direction in Fig. 16 (route indicated with a red arrow) is indicated with the red line in Fig. 17.

In this case, $\omega=v k_{x}$. Similarly, the dispersion relation along the blue arrow is indicated with the blue line in Fig. 17. In this case, $\omega=v k_{2}$ and $k_{2}$ is

$$
k_{2}=\sqrt{k_{y}^{2}+\left(\frac{2 \pi}{a}\right)^{2}} .
$$

This line has a small slope owing to the square root nature. To consider multiple diffractions, we simply shift the band by an integer multiple of an elementary reciprocal lattice vector and plot within the range of the first Brillouin zone.
[1] R. P. Feynman, QED : The Strange Theory of Light and Matter (Princeton University Press, Princeton, NJ, 1987).

[2] E. Yablonovitch, Inhibited Spontaneous Emission in Solid-State Physics and Electronics, Phys. Rev. Lett. 58, 2059 (1987).

[3] S. John, Strong Localization of Photons in Certain Disordered Dielectric Superlattices, Phys. Rev. Lett. 58, 2486 (1987).

[4] J. D. Joannopoulos, R. D. Meade, and J. N. Winn, Photonic Crystals, Molding the Flow of Light (Princeton University Press, Princeton, NJ, 1995).

[5] K. Sakoda, Optical Properties of Photonic Crystals (SpringerVerlag, Berlin, Heidelberg, 2005).

[6] M. Imada, S. Noda, A. Chutinan, T. Tokuda, M. Murata, and G. Sasaki, Coherent two-dimensional lasing action in surfaceemitting laser with triangular-lattice photonic crystal structure, Appl. Phys. Lett. 75, 316 (1999).

[7] M. Meier, A. Mekis, A. Dodabalapur, A. Timko, R. E. Slusher, J. D. Joannopoulos, and O. Nalamasu, Laser action from two- dimensional distributed feedback in photonic crystals, Appl. Phys. Lett. 74, 7 (1999).

[8] K. Inoue, M. Sasada, J. Kawamata, K. Sakoda, and J. W. Haus, A two-dimensional photonic crystal laser, Jpn. J. Appl. Phys. 38, L157 (1999).

[9] M. Meier, A. Dodabalapur, J. A. Rogers, R. E. Slusher, A. Mekis, A. Timko, C. A. Murray, R. Ruel, and O. Nalamasu, Emission characteristics of two-dimensional organic photonic crystal lasers fabricated by replica molding, J. Appl. Phys. 86, 3502 (1999).

[10] M. Notomi, H. Suzuki, and T. Tamamura, Directional lasing oscillation of two-dimensional organic photonic crystal lasers at several photonic band gaps, Appl. Phys. Lett. 78, 1325 (2001).

[11] M. Imada, A. Chutinan, S. Noda, and M. Mochizuki, Multidirectionally distributed feedback photonic crystal lasers, Phys. Rev. B 65, 195306 (2002). 
[12] D. Ohnishi, T. Okano, M. Imada, and S. Noda, Room temperature continuous wave operation of a surface-emitting two-dimensional photonic crystal diode laser, Opt. Express 12, 1562 (2004).

[13] K. Hirose, Y. Liang, Y. Kurosaka, T. Sugiyama A.Watanabe, and S. Noda, Watt-class high-power, high-beam-quality photonic-crystal lasers, Nat. Photonics 8, 406 (2014).

[14] M. Yoshida, M. D. Zoysa, K. Ishizaki, Y. Tanaka, M. Kawasaki, R. Hatsuda, B. Song, J. Gelleta, and S. Noda, Double-lattice photonic-crystal resonators enabling high-brightness semiconductor lasers with symmetric narrow-divergence beams, Nat. Mater. 18, 121 (2019).

[15] M. Notomi, T. Tamamura, Y. Ohtera, O. Hanaizumi, and S. Kawakami, Direct visualization of photonic band structure for three-dimensional photonic crystals, Phys. Rev. B 61, 7165 (2000).

[16] K. Sakai, E. Miyai, T. Sakaguchi, D. Ohnishi, T. Okano, and S. Noda, Lasing band-edge identification for a surface-emitting photonic crystal laser, IEEE J. Sel. Areas Commun. 23, 1335 (2005).

[17] N. Le Thomas, R. Houdré, D. M. Beggs, and T. F. Krauss, Fourier space imaging of light localization at a photonic bandedge located below the light cone, Phys. Rev. B 79, 033305 (2009).

[18] N.-V.-Q. Tran, S. Combrié, and A. De Rossi, Directive emission from high-Q photonic crystal cavities through band folding, Phys. Rev. B 79, 041101(R) (2009).

[19] S. L. Portalupi, M. Galli, C. Reardon, T. F. Krauss, L. O'Faolain, L. C. Andreani, and D. Gerace, Planar photonic crystal cavities with far-field optimization for high coupling efficiency and quality factor, Opt. Express 18, 16064 (2010).

[20] Y. Kurosaka, K. Hirose, A. Watanabe, T. Sugiyama, Y. Liang, and S. Noda, Effects of non-lasing band in two-dimensional photonic-crystal lasers clarified using omnidirectional band structure, Opt. Express 20, 21773 (2012).

[21] M. Minkov, V. Savona, and D. Gerace, Gallium nitride L3 photonic crystal cavities with an average quality factor of 16 900 in the near infrared, Appl. Phys. Lett. 111, 131104 (2017).

[22] M. S. Mohamed, A. Simbula, J.-F. Carlin, M. Minkov, D. Gerace, V. Savona, N. Grandjean, M. Galli, and R. Houdré, Efficient continuous-wave nonlinear frequency conversion in high-Q gallium nitride photonic crystal cavities on silicon, APL Photon. 2, 031301 (2017)

[23] J. Wang, A. Chen, M. Zhao, W. Liu, Y. Zhang, X. Liu, L. Shi, and $\mathrm{J} . \mathrm{Zi}$, Observation of optical states below the light cone with compound lattices, OSA Continuum 2, 1844 (2019).
[24] Y. Kurosaka, S. Iwahashi, Y. Liang, K. Sakai, E. Miyai, W. Kunishi, D. Ohnishi, and S. Noda, On-chip beamsteering photonic-crystal lasers, Nat. Photonics 4, 447 (2010).

[25] Y. Kurosaka, S. Iwahashi, K. Sakai, E. Miyai, W. Kunishi, D. Ohnishi, and S. Noda, Band structure observation of 2D photonic crystal with various $\mathrm{V}$-shaped air-hole arrangements, IEICE Electron. Express 6, 966 (2009).

[26] D. Levine and P. J. Steinhardt, Quasicrystals: A New Class of Ordered Structures, Phys. Rev. Lett. 53, 2477 (1984).

[27] M. V. Jaric, Diffraction from quasicrystals: Geometric structure factor, Phys. Rev. B 34, 4685 (1986)

[28] M. Notomi, H. Suzuki, T. Tamamura, and K. Edagawa, Lasing Action due to the Two-Dimensional Quasiperiodicity of Photonic Quasicrystals with a Penrose Lattice, Phys. Rev. Lett. 92, 123906 (2004).

[29] Z. V. Vardeny, A. Nahata, and A. Agrawal, Optics of photonic quasicrystals, Nat. Photonics 7, 177 (2013).

[30] Y. Kurosaka, K. Hirose, T. Sugiyama, Y. Takiguchi, and Y. Nomoto, Phase-modulating lasers toward on-chip integration, Sci. Rep. 6, 30138 (2016).

[31] Y. Takiguchi, K. Hirose, T. Sugiyama, Y. Nomoto, S. Uenoyama, and Y. Kurosaka, Principle of beam generation in on-chip 2D beam pattern projecting lasers, Opt. Express 26, 10787 (2018).

[32] K. Hirose, Y. Takiguchi, T. Sugiyama, Y. Nomoto, S. Uenoyama, and Y. Kurosaka, Principle of beam generation in on-chip 2D beam pattern projecting lasers, Opt. Express 26, 29854 (2018).

[33] K. Hirose, H. Kamei, T. Sugiyama, and Y. Kurosaka, $200 \times$ $200 \mu \mathrm{m}^{2}$ structured light source, Opt. Express 28, 37307 (2020).

[34] J. Goodman, Introduction to Fourier Optics, 3rd ed. (Roberts \& Company, Greenwood Village, CO, 2005).

[35] B. R. Brown and A. W. Lohmann, Complex spatial filtering with binary masks, Appl. Opt. 5, 967 (1966).

[36] R. W. Gerchberg and W. O. Saxton, A practical algorithm for the determination of phase from image and diffraction plane pictures, Optik 35, 237 (1972).

[37] S. Noda, K. Kitamura, T. Okino, D. Yasuda, and Y. Tanaka, Photonic-crystal surface-emitting lasers: Review and introduction of modulated-photonic crystals, IEEE J. Sel. Top. Quantum Electron. 23, 4900107 (2017).

[38] iPMSEL, https://www.hamamatsu.com/jp/en/our-company/ about-crl/optical-materials/ipmsel.html. 